\title{
Research on Fatigue Damage and Affecting Factors of Defected Rock Mass Based on Ultrasonic Wave Velocity
}

\author{
Run LI, Yasi WANG, Fengfei FU
}

\begin{abstract}
In this paper the fatigue-loading test of defected rock-like specimen has been carried out by electro-hydraulic servo fatigue testing machine. At the same time, the ultrasonic data has been collected and analyzed by the digital ultrasonic instrument. The fatigue damage and influencing factors of the defective rock mass under dynamic load are studied. According to the experimental research and analysis, this paper chooses to define the damage variable by ultrasonic velocity, and the inverse function of the Logistic equation was used to describe the evolution curve of fatigue damage sample. The experimental data fitting results show that the damage model and the experimental data fit well. In addition, this paper analyzes the main influencing factors of fatigue damage in the test. The initial damage represents the damage state before the sample is cyclically loaded. Different initial damages have a significant effect on the fatigue life of the sample. In the case the initial damage is substantially the same, the upper limit stress is larger, the fatigue life of the sample is shorter, and conversely, the fatigue life is longer.
\end{abstract}

Keywords: affecting factors; defected rock; dynamic loading; fatigue damage; ultrasonic wave velocity

\section{INTRODUCTION}

As a natural structure, the rock mass has weak structural surfaces with a large number of irregular cracks, joints, holes, interlayers and so on. The existence of these weak structural surfaces has a great influence on the mechanical properties of the rock mass. For the slope of rock mass along the road and railway, the geological environment is complex, and the rock mass has many weak structural planes. Under the long-term influence of traffic dynamic load, the rock mass has fatigue damage and the mechanical properties are degraded. There are major hidden dangers in the long-term safety and stability.

Due to the differences in the formation process, the defective rock mass is difficult to sample, the test influence conditions are complex, and the theoretical evaluation involves many variables. Its mechanical property and failure mechanism have always been the subject of research by geotechnical researchers. In recent years, with the improvement of the safety and durability requirements of rock mass engineering, many scholars at home and abroad have carried out a large number of theoretical and experimental studies on the defective rock mass.

M. Prudencio et al. [1] studied the rock physical model with non-persistent joints. The study found that the joint geometry, the principal stress direction, and the ratio of the intermediate stress to the compressive strength of the intact material $\left(\sigma^{2} / \sigma_{\mathrm{c}}\right)$ determine the failure mode and maximum strength of the specimen. TIEN YONG MING et al. [2] obtained the unrolled images of the rock sample at different stress levels in the uniaxial compression test through the rotary scanner, by analyzing the expanded images; the mechanism of the destruction of transversely isotropic rocks is studied. Mingxing Liu et al. [3] used cyclic triaxial test to study the mechanical behavior of artificial jointed rock specimens under different stress amplitudes and different confining pressures, and the evolutionary characteristics of the residual axial strain and residual dilatancy with loading cycles, as well as the evolution law of damages, are determined by the stress ratios. Yi Liu et al. [4] studied the fatigue mechanical properties of jointed rock models under different cyclic conditions and the experimental results reveal the influence of the cyclic load parameters on the mechanical properties of jointed rock models. Debasis Deb et al. [5] used the extended finite element method (XFEM) to numerically simulate the discontinuous rock mass, and the calculated analytical results agree well with the experimental results. Zhang, X. P. et al. [6] studied the crack initiation, propagation, and coalescence of rock-like materials with two defects under uniaxial compression by Bonded-Particle Model. WASANTHA P L P et al. [7] used numerical simulation to study the effect of crack geometry on rock mechanical properties under uniaxial compression test, and the numerical simulation results agree well with the experimental results.

Due to the existence of irregularly distributed discontinuous structural planes such as joints and cracks in the rock mass, the rock mass exhibits anisotropic characteristics, and the ultrasonic wave carries extremely rich information when it passes through the rock mass. It is a comprehensive reflection of the internal structural features of the rock. Compared with the static method, the ultrasonic testing technology is not only simple, reliable, and economical, but also it has no damage to the test rock sample or rock mass, and it has received extensive attention from geotechnical workers at home and abroad.

M. Darot et al. [8] studied the changes in acoustic wave velocity and permeability during pressure cycling of granite samples with hot cracks at high temperatures. Song et al. [9] obtained the dynamic elastic modulus of the rock by testing the acoustic wave velocity of the layered rock and analyzing the measurement results. McCann et al. [10] used a certain ultrasonic frequency, pressure, and temperature to study the compression wave velocity, shear wave velocity, longitudinal wave, and shear wave attenuation characteristics of sandstone and limestone samples. Cengiz et al. [11] studied the ultrasonic propagation of ultrasonic waves in parallel joints and joints in different directions by testing marble samples. By quantifying the change of ultrasonic wave velocity to reflect the change of rock microstructure caused by the action of $\mathrm{CO}_{2}$ and water, Adam et al. [12] established the relationship between elastic wave velocity and rock microstructure change.

At present, the research on the mechanical properties and engineering characteristics of the defective rock mass is mostly concentrated under static load conditions. Compared with other materials, the research on the fatigue 
of damaged rock mass is still limited. It is difficult to sample the natural rock mass, have large individual differences in rock samples, and have complicated test conditions. In order to effectively and specifically study the influence of weak structures on rock mass, this paper studies the fatigue damage of defective rock mass under dynamic load by means of model test, and this paper analyzes the influencing factors of fatigue damage. The results of the study help to scientifically evaluate rock mass stability, predict rock fatigue life, and fatigue design.

\section{TEST OVERVIEW}

\subsection{Model Materials and Sample Preparation}

The test model samples are made of barite powder, river sand (particle size less than $0.5 \mathrm{~mm}$ ), composite Portland cement, model gypsum, borax solution (concentration: 1\%). The mass ratio of materials is barite powder: river sand: cement: gypsum: borax solution $=42.0$ 21.5: $8.5: 12.7: 15.3$. The mold has been assembled from cast iron and fixed by card slot and screw. The slit plate slot has been arranged on the bottom plate to facilitate the installation of cracks and ensure the accurate positioning of the crack. The sample size is $70 \times 70 \times 140 \mathrm{~mm}$, the crack is set by pre-inserted iron piece, the crack length is $20 \mathrm{~mm}$, the crack angle is set to $0^{\circ}, 30^{\circ}, 45^{\circ}, 60^{\circ}, 90^{\circ}$. The thin iron sheet is pulled out after the sample is maintained 6 hours. The characteristic of heat expansion during solidification of the model material is used to make the crack surface close as much as possible. After removing the mould, the samples are placed in the dry and ventilated place and dried naturally for 20 days. Through strict control of the sample preparation process, the finished samples are weighed one by one, and the overweight or under-thickness samples are removed to ensure the uniformity of the samples and reduce the influence of the dispersion on the test results. Each group has 3 samples. The mold and crack samples are shown in Fig. 1.

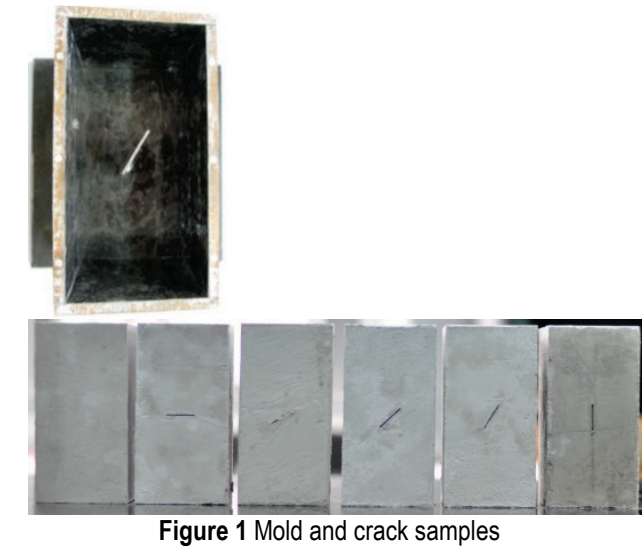

\subsection{Test Equipment and Loading Conditions}

The test loading system is an electro-hydraulic servo fatigue testing machine, which adopts equal-amplitude load control mode, and the loading waveform adopts a sine wave with a loading frequency of $5 \mathrm{~Hz}$. According to the uniaxial compressive strength of the sample, the control load amplitude ranges from 1.9 to $2.4 \mathrm{MPa}$. In the process of cyclic loading of the sample, real-time monitoring and acquisition of ultrasonic data is performed by means of a digital ultrasonic instrument. The schematic diagram of ultrasonic measurement is shown in Fig. 2.

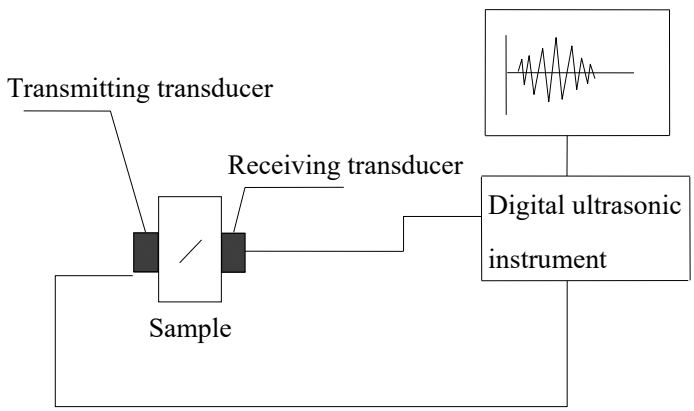

Figure 2 Schematic diagram of ultrasonic measurement

\section{FATIGUE DAMAGE MODEL \\ 3.1 Selection of Damage Variable}

The damage variable means the measure level of fatigue damage inside the material. For rock materials, it can be intuitively understood as the percentage of microcracks and micro-pores in the material, which can reflect the process of fatigue damage development. Depending on the research perspective, there are many options for damage variables. From the macroscopic view, elastic modulus, dissipative energy, ultrasonic velocity, acoustic emissivity can be selected. From the microscopic view, crack density, number of pores can be selected. For a reasonable fatigue cumulative damage theory, the physical meaning of the damage variable should be clear, the measurement is simple and convenient, and the damage evolution law is consistent with the experimental data [13].

When ultrasonic waves propagate in rock mass by certain defects in the rock mass, ultrasonic waves will produce various propagation phenomena such as refraction, reflection, transmission, scattering, and diffraction. The degree of energy attenuation of ultrasonic waves during propagation is affected by the crack angle, and the displayed wave speed, amplitude, frequency will also be different.

The ultrasonic velocity of the model specimens at the time of unloading has been collected and counted, and the relation curve $V-\alpha$ between the ultrasonic velocity and the fractured dip is made. The ultrasonic velocity is normalized, and the ultrasonic velocity $V_{0}$ of the intact specimen is used as the reference parameter. The relation curve $\bar{V}-\alpha$ between the wave velocity ratio and the fractured dip is made. The wave velocity ratio is $\bar{V}=V_{i} / V_{0}$ ( $V_{i}$ means the ultrasonic velocity of the sample for each crack angle). The relation curves between the ultrasonic velocity and the fractured dip are shown in Fig. 3.

Fig. 3 shows:

(1) The longitudinal wave velocity of the model samples with the preset crack is lower than that of the intact sample.

(2) The longitudinal wave velocity of the model sample shows a significant decrease trend with the increase of the crackdip angle.

(3) The longitudinal wave velocity of model samples with crack dip angles of $45^{\circ}, 60^{\circ}$ and $90^{\circ}$ decreased more than those of model samples with $0^{\circ}$ and $30^{\circ}$. 


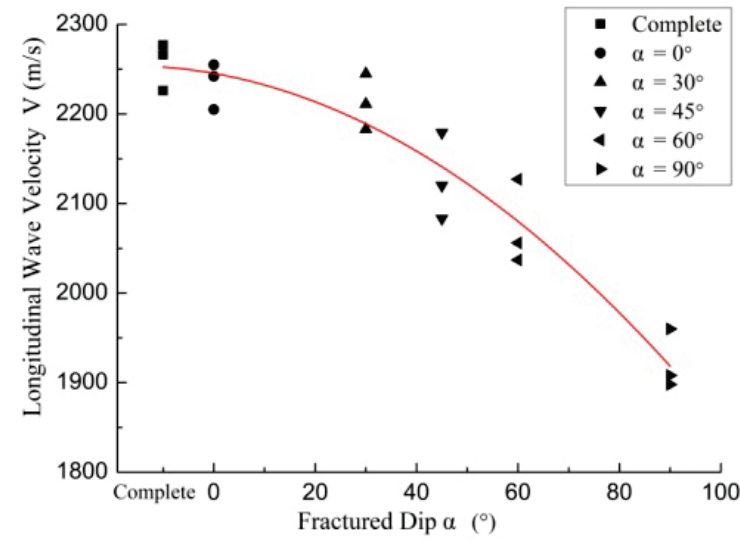

(a) $V-\alpha$

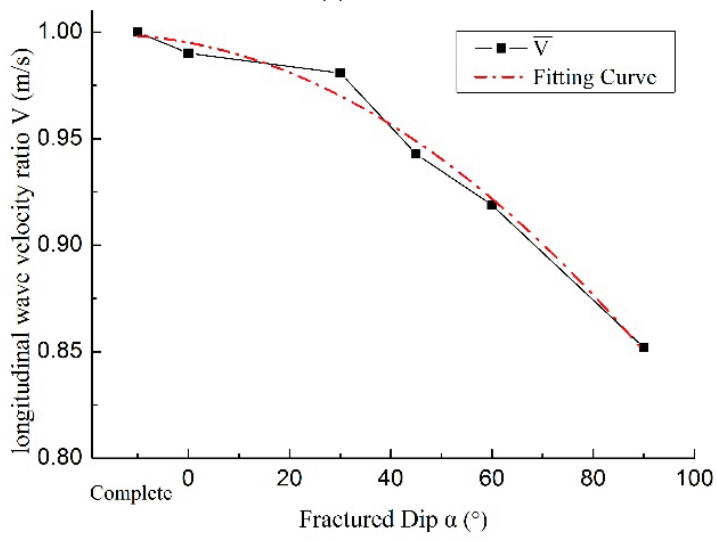

(b) $\bar{V}-\alpha$

Figure 3 Relation curve between longitudinal wave velocity and fractured dip of model specimens when not loaded

The reason for the above situation can be analyzed from the perspective influence of the crack size on the ultrasonic velocity. For the crack with a certain width, as the crack angle increases, the projection width of the crack increases in the direction perpendicular to the ultrasonic wave. The projection width of the crack dip angles $0^{\circ}$ is the smallest, and that of $90^{\circ}$ is the largest. With the increase of crack dip angle, the width of interface which hinders the propagation of ultrasonic wave becomes larger, and the ultrasonic energy is more dissipated at the defect, which has an effect on the ultrasonic speed.

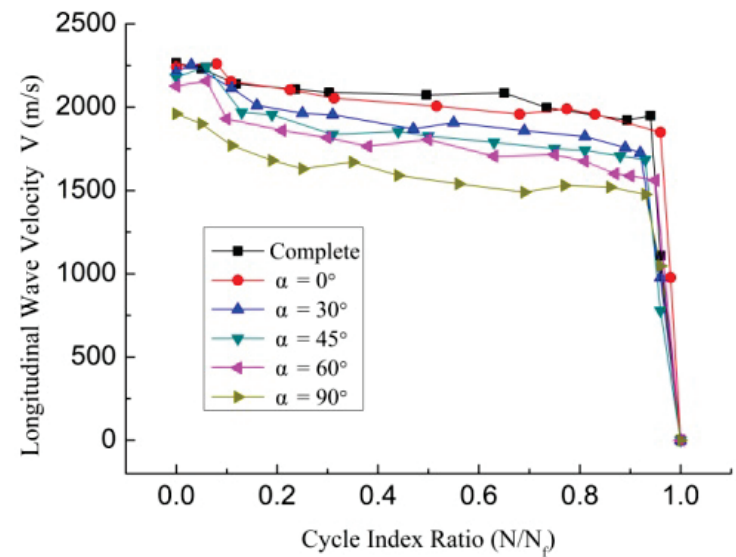

Figure 4 Ultrasonic velocity decay pattern of model specimens under dynamic load

Considering that the longitudinal wave velocity of the model sample has a certain relationship with the dip angle of the crack, this paper uses the digital ultrasonic instrument to test the ultrasonic velocity at a certain number of cycles during the cyclic loading of the model sample until the sample is destroyed. The relationship between the longitudinal wave velocity and the cycle index ratio of the model sample under dynamic load is shown in Fig. 4. Here, the cycle index ratio $N / N_{f}$ refers to the ratio of the cycle number $N$ under a given cyclic load to the corresponding fatigue life $N_{f}$.

The experimental study found that the ultrasonic velocity of the sample has obviously attenuated during cyclic loading, which is sensitive to changes with damage, and the change is also stable, and has obvious physical meaning and engineering applicability. Therefore, the ultrasonic velocity is used to define the damage variable in this paper.

When the acoustic frequency is higher than $20 \mathrm{kHz}$, the relationship between the longitudinal wave velocity of the rock and the elastic constant and density of the material is as follows [14].

$$
v_{p}=\sqrt{\frac{G}{\rho}}=\sqrt{\frac{E}{2 \rho(1+v)}}
$$

Where: $\rho$ is the material density; $E, G$ and $v$ are the material elastic constants.

The longitudinal wave velocity $\tilde{v}_{p}$ of the damaged material has a similar relationship to the effective elastic constants $\tilde{E}, \tilde{G}, \tilde{v}$ and density $\tilde{\rho}$ of the damaged material:

$$
\tilde{v}_{p}=\sqrt{\frac{\tilde{G}}{\tilde{\rho}}}=\sqrt{\frac{\tilde{E}}{2 \tilde{\rho}(1+\tilde{v})}}
$$

In the process of material damage, the density change is usually small, and the change can be neglected. The damage variables represented by the changes of the longitudinal wave velocity, which is determined by Eqs. (1) and (2) are as follows.

$$
D=1-\frac{\tilde{G}}{G}=1-\frac{\tilde{v}_{p}^{2}}{v_{p}^{2}}
$$

Because the ultrasonic velocity of rock is closely related to rock density, elastic constant and internal microcrack, the internal structure of the material will change significantly under dynamic load, such as the formation, expansion, and penetration of micro-cracks, local collapse inside the material, and so on. The nondestructive testing instrument (such as: ultrasonic instrument) can easily reflect the deterioration of the material, so the damage variable given by Eq. (3) can comprehensively reflect the deterioration degree of each parameter of the rock.

For the model specimens with different crack angles, if the ultrasonic velocity $v_{P 0}$ of the model specimen in the formula (3) is replaced by the corresponding unloaded model $\tilde{v}_{P}$, and the ultrasonic velocity $v_{P}$ at the time when the intact model specimen is not loaded, the initial damage variables of the model specimens with different crack angles are obtained as follows: 


$$
D_{0}=1-\frac{v_{P 0}^{2}}{v_{P}^{2}}
$$

\subsection{Fatigue Damage Evolution Pattern}

For typical samples with different crack angles, their respective initial damage $D_{0}$ has been calculated by Eq. (4), as shown in Tab. 1.

\begin{tabular}{|c|c|c|c|c|c|c|}
\hline $\begin{array}{c}\text { Track angle 1 } \\
(\alpha)\end{array}$ & Intact & $0^{\circ}$ & $30^{\circ}$ & $45^{\circ}$ & $60^{\circ}$ & $90^{\circ}$ \\
\hline $\begin{array}{c}\text { Initial damage of the model sample } D_{0} \\
\text { damage }\left(D_{0}\right)\end{array}$ & 0 & 0.02107 & 0.04796 & 0.07531 & 0.11892 & 0.20184 \\
\hline
\end{tabular}

The ultrasonic velocity measured during the cyclic loading test of each sample under the same upper limit stress $S_{\max }=2.1 \mathrm{MPa}$ is substituted into Eq. (3), and the corresponding damage variable $D$ is calculated. Taking the damage variable $D$ as the ordinate and the cycle index ratio $N / N_{f}$ as the abscissa, the fatigue damage evolution curve of the model specimen is obtained, as shown in Fig. 5.

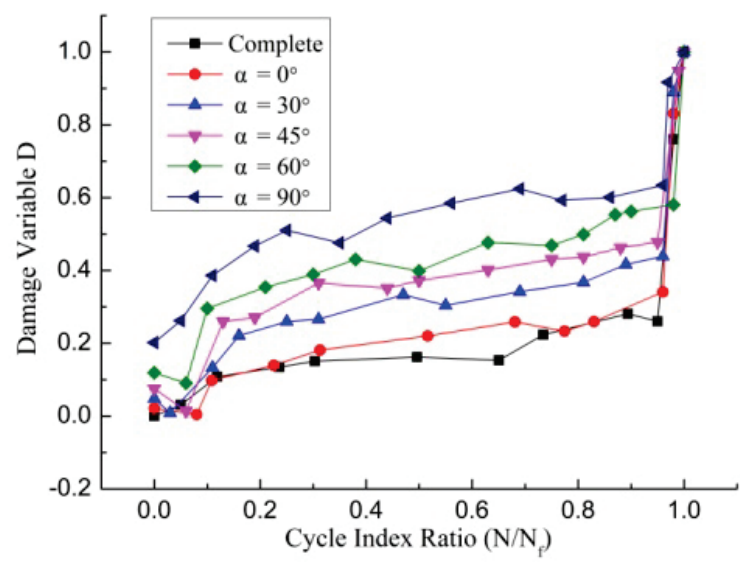

Figure 5 Evolution pattern of fatigue damage of model specimens

Fig. 5 shows:

(1) The evolution pattern of fatigue damage specimens shows a clear three-stage trend. In the first stage, the damage rate is relatively large, and it develops to a relatively stable level, which accounts for $10 \%$ to $20 \%$ of the total fatigue life. The second stage is the stage of slow accumulation of fatigue damage, accounting for $75-85 \%$ of the total fatigue life. The third stage of the damage increased sharply, the sample quickly reached the damage, and this stage accounted for about $5 \%$ of the total fatigue life.

(2) From the overall trend, as the cyclic loading progresses, the fatigue damage of the sample is generally increasing.

(3) In the first stage, most samples showed different degrees of negative damage growth, because in the initial stage of cyclic loading, the preset internal cracks, micro-cracks, pores, and so on were compacted and the wave velocity was increased.

\subsection{Fatigue Damage Model}

In order to describe the fatigue damage evolution of model specimens better, it is necessary to establish a suitable mathematical model to reflect the variation of fatigue damage of specimens. It can be seen from Fig. 5, the fatigue damage evolution pattern of the sample reflected by the damage variable defined by the ultrasonic velocity defines that the three-stage characteristics of the fatigue damage evolution pattern of the model specimen are obvious, and the overall trend of the inverted S-type is increasing. Therefore, the established fatigue cumulative damage equation also needs to reflect the three-stage variation of this inverted S-type. By fitting the test data, the results show that the inverted S-type three-stage curve can be expressed by the inverse function of the Logistic equation. The inverse function of the Logistic equation is as follows:

$y=a-b \cdot \ln \left(\frac{k}{x}-p\right)$

Applied to the fatigue damage evolution of the specimen, it can be rewritten as follows:

$D=a-b \cdot \ln \left(\frac{k}{n}-p\right)$

Where: $n$ is an independent variable, according to the test in this paper, $n$ takes the cycle index ratio; $D$ is the dependent variable, refers to the cumulative fatigue damage of the sample; $a, b, k$ and $p$ are the fitting parameters.

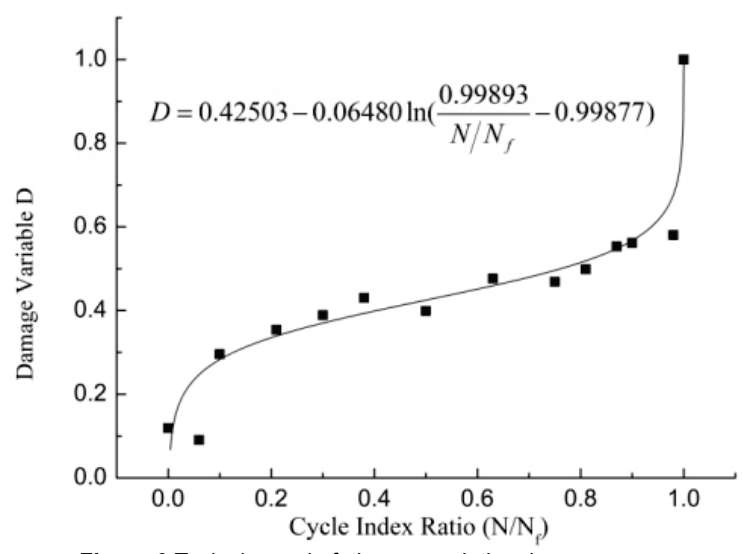

Figure 6 Typical sample fatigue cumulative damage curve

By adjusting the values of the fitting parameters $a, b$, $k$ and $p$ the fatigue cumulative damage evolution curve which is well fitted to the experimental results can be obtained. The fitting curve of the typical model sample is shown in Fig. 6.

\subsection{Test Data Fitting Verification}

The data obtained from the test is curve-fitted according to the fatigue cumulative damage model of Eq. (6), and the degree of fitting of the curve is determined by the corresponding correlation coefficient $R^{2}$.

The results of the fitting curve of this test data are shown in Fig. 7.

Fig. 7 shows that the inverse function form of the Logistic equation can better describe the inverse S-type 
three-stage pattern of sample fatigue damage evolution. From the fitting results of the experimental data, the correlation coefficients $R^{2}$ are all above 0.81 , indicating that the damage model and the experimental data fit well.

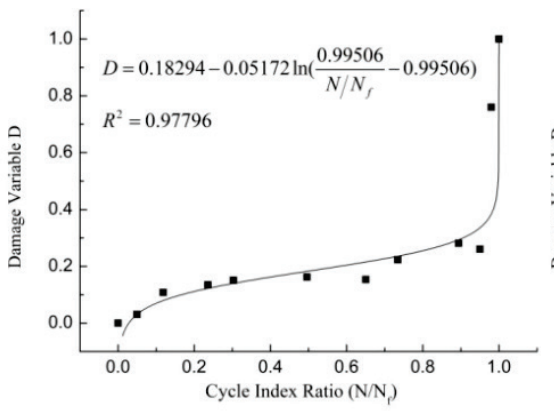

(a) Intact sample

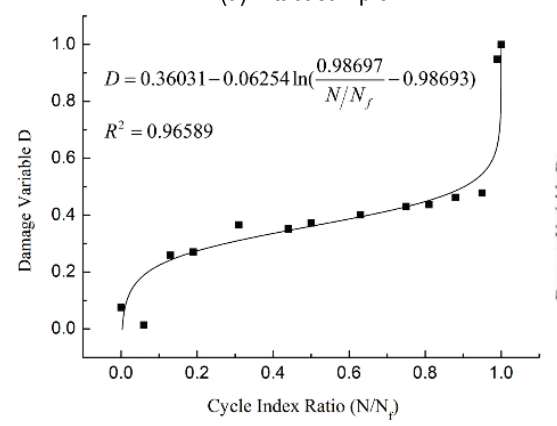

(d) $\alpha=45^{\circ}$

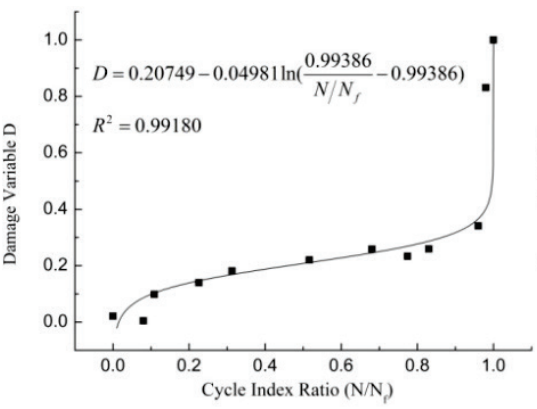

(b) $\alpha=0^{\circ}$

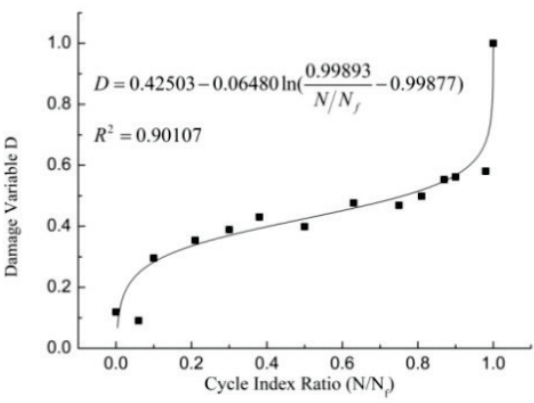

(e) $\alpha=60^{\circ}$

Figure 7 Fit curve of damage model and test data

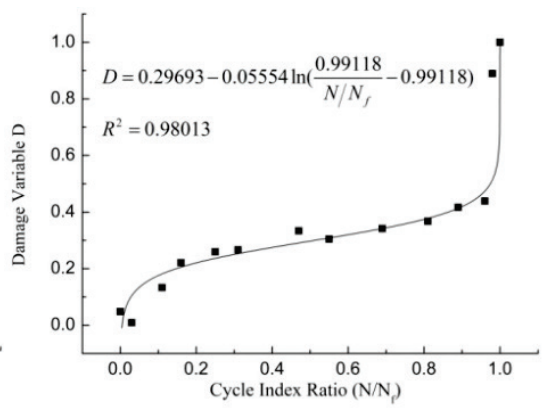

(c) $\alpha=30^{\circ}$

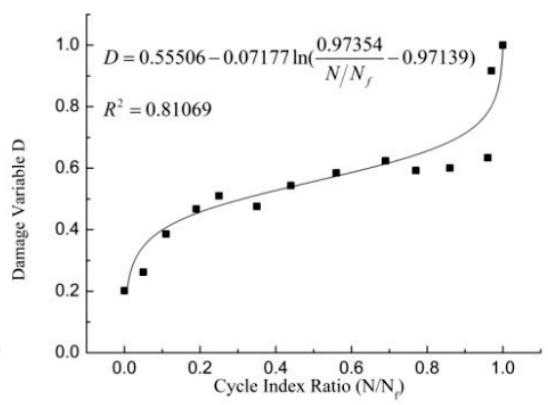

(f) $\alpha=90^{\circ}$

\section{ANALYSIS OF FACTORS AFFECTING FATIGUE DAMAGE}

The factors affecting the cumulative damage evolution of rock fatigue can be roughly divided into two aspects. On the one hand is the composition and structure of the rock itself. On the other hand is the environmental condition in which the rock is located. The composition and structure of the rock include the materials that make up the rock (the material of the rock itself, the filling of the weak structural surface, etc.), the quantity and distribution of the defects in the rock, and so on. The environmental conditions of the rock include the load conditions of the rock (static load, dynamic load), environmental and climatic conditions, ambient temperature conditions, and so on. Starting from the test, this paper mainly considers the influence of initial damage and upper limit stress on the cumulative damage evolution of the sample.

\subsection{Effect of Initial Damage}

There are defects such as joints, cracks, micro-cracks and holes in natural rocks. The number, distribution, and angle of formation of these defects cause different degrees of initial damage to the rock. In this test, different initial cracks are simulated by presenting the cracks at different angles to the model specimens. The initial damage represents the damage state before the specimen is cyclically loaded.

The cumulative damage fitting curves and fitting parameters of Fig. 7 are analyzed, and it was found that different initial damages had certain influence on the parameters $a$ and $b$. The initial damage $D_{0}$ of the samples and the parameter values $a$ and $b$ are shown in Tab. 2 .
Table 2 Initial damage $D_{0}$ of model specimens and parameter values $a$ and $b$

\begin{tabular}{|c|c|c|c|}
\hline Crack angle & Initial damage $D_{0}$ & Parameter $a$ & Parameter $b$ \\
\hline Intact & 0 & 0.18294 & 0.05172 \\
\hline $0^{\circ}$ & 0.02107 & 0.20749 & 0.04981 \\
\hline $30^{\circ}$ & 0.04796 & 0.29693 & 0.05554 \\
\hline $45^{\circ}$ & 0.07531 & 0.36031 & 0.06254 \\
\hline $60^{\circ}$ & 0.11892 & 0.42503 & 0.06480 \\
\hline $90^{\circ}$ & 0.20184 & 0.55506 & 0.07177 \\
\hline
\end{tabular}

Tab. 2 shows:

(1) The different values of the parameter $a$ mainly affect the intercept of the curve on the ordinate, and this intercept reflects the initial damage of the sample, as the initial damage $D_{0}$ increases, the parameter $a$ also increases.

(2) The parameter $b$ reflects the slope of the second stage of cumulative damage, this stage is the most important stage for the fatigue life.

(3) The value of parameter $b$ increases with the increase of initial damage of cracked specimens.

However, the size of the initial damage is not the only factor affecting the slope of the second stage of cumulative damage. The difference in the angle and the number of cracks will also cause the difference of crack formation, expansion, and penetration during the development of fatigue damage in the specimen. The slope of the second stage will also be affected.

\subsection{Influence of Upper Limit Stress}

The upper limit stress has an important influence on the fatigue life of the specimen. The typical specimens are cyclically loaded with different upper limit stresses. The fatigue damage evolution pattern of the specimens is shown in Fig. 8. 


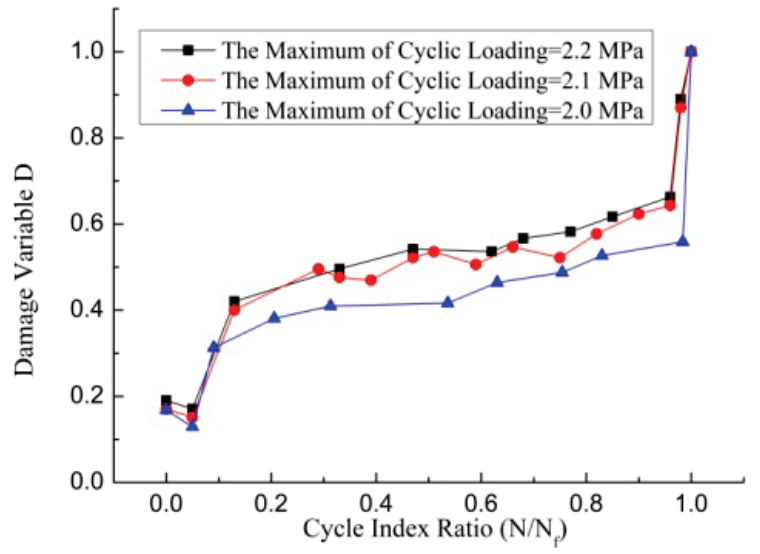

Figure 8 Typical fatigue damage evolution pattern of specimens

Fig. 8 shows:

(1) When the initial damage is about the same, the larger the upper limit stress is, the larger the fatigue damage value of the first stage of the specimen will be, and the smaller the upper limit stress is, the smaller the fatigue damage value of the first stage of the specimen will be.

(2) When the upper limit stress is larger, the ratio of the first stage and the third stage to the total number of cycles is also larger, the corresponding second stage accounts for a small proportion of the total cycle number.

(3) When the upper limit stress is small, the ratio of the first stage and the third stage to the total number of cycles is also small, and the corresponding second stage accounts for a large proportion of the total cycle number.

(4) The second stage of fatigue damage is the main stage of the fatigue life of the specimen, and it can be judged that when the upper limit stress is large, the fatigue life of the specimen is short, conversely, the fatigue life of the specimen is long.

The fatigue damage evolution pattern of the specimen under different upper stress levels was fitted. The results are shown in Fig. 9. As shown in Fig. 9, the value of the fitting curve parameter $b$ increases with the increase of the upper limit stress, and the parameter $b$ represents the second stage slope of the fitting curve, indicating that the slope of the second stage of fatigue damage accumulation process of the sample increases with the increase of the upper limit stress. The slope of the second stage becomes larger, the damage rate of the sample increases in the second stage, and the damage of the second stage of the sample increases.

Here, the fatigue threshold of the material needs to be considered. The fatigue threshold is the stress threshold for the fatigue failure of the rock. Ge Xiurun [15] proposed that whether the rock was damaged was related to the stress threshold, and that the rock fatigue threshold was close to the conventional yield value. The upper limit stress ratio range of this test is $0.76-0.90$. It is proved by experiments that this range is more suitable.

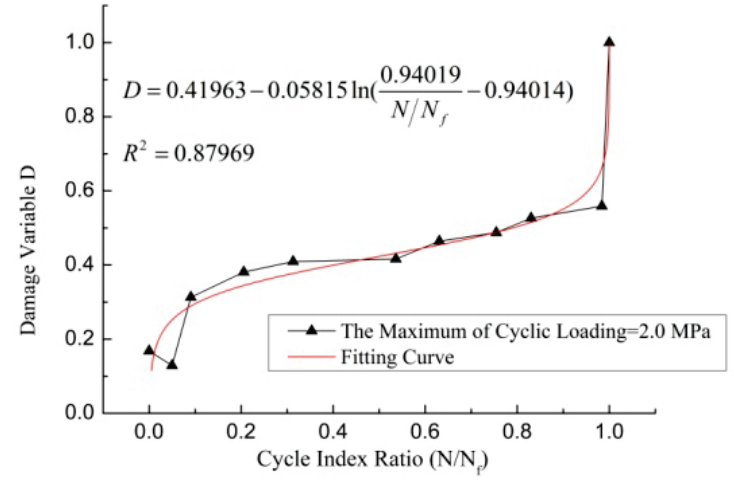

(a) Upper limit stress 2.0MPa

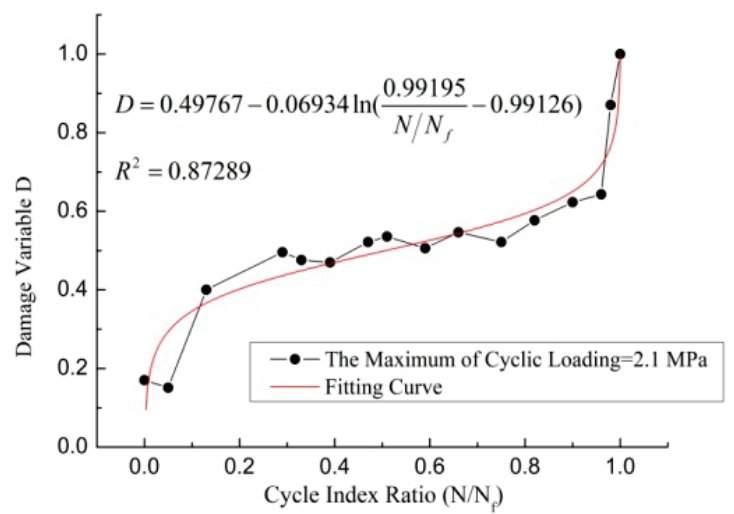

(b) Upper limit stress $2.1 \mathrm{MPa}$

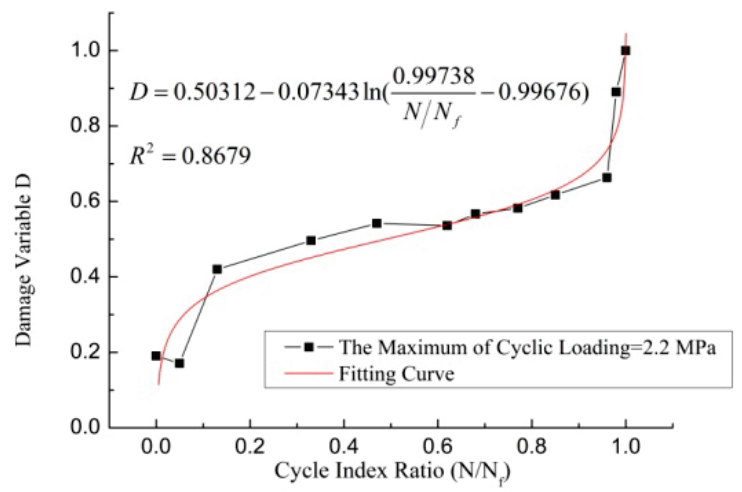

(c) Upper limit stress $2.2 \mathrm{MPa}$

Figure 9 Damage evolution pattern fitting curve of typical specimens with different upper limit stresses

\section{CONCLUSION}

In this paper, the intact and fracture model samples are cyclically loaded, and the acoustic wave data have been collected by the digital ultrasonic instrument. Based on the analysis of acoustic wave data, the damage variable has been selected and the appropriate fatigue cumulative damage model is given. In addition, this paper analyzes and studies the factors affecting fatigue damage. The main conclusions are as follows:

(1) The ultrasonic velocity of the sample is sensitive to the change of fatigue damage, and it is stable. The test is convenient and the physical meaning is clear. The ultrasonic variable can be selected to define the damage variable.

(2) The fatigue damage evolution pattern of the specimens with the damage variable definition of the ultrasonic velocity is increasing overall, and has obvious threestage characteristics.

(3) The inverted S-type three-stage curve of the fatigue 
damage evolution of the specimen can be described by the inverse function of the Logistic equation. The correlation coefficient $R^{2}$ of the test data fitting results is above 0.81, and the damage model and the experimental data are well fitted.

(4) The initial damage represents the damage state before the sample is cyclically loaded, and different initial damages have a significant influence on the fatigue life of the sample.

(5) The upper limit stress is the primary factor affecting the fatigue life of the sample. In the case where the initial damage is approximately the same, the upper limit stress is larger, the fatigue life of the sample is shorter, and conversely, the fatigue life is longer.

\section{Acknowledgements}

This work was kindly supported by Natural Science Foundation of Fujian Province (No. 2015J05077), Natural Science Foundation of Fujian Province (No. 2016J01206), Young and Middle-aged Education Scientific Research Project of Fujian Province (JA15533) and China Scholarship Council (CSC, No. 201608350057).

\section{REFERENCES}

[1] Prudencio, M. \& Van Sint Jan, M. (2007). Strength and failure modes of rock mass models with non-persistent joints. Int. J. Rock Mech. Min. Sci., 44, 890-902. https://doi.org/10.1016/j.jijmms.2007.01.005

[2] Tien Yong Ming, Kuo Ming Chuan, \& Juang Charng Hsein. (2006). An experimental investigation of the failure mechanism of simulated transversely isotropic rock. International Journal of Rock Mechanics and Mining Sciences, 43(8), 1163-1181. https://doi.org/10.1016/j.jirmms.2006.03.011

[3] Mingxing Liu \& Enlong Liu. (2017). Dynamic mechanical properties of artificial jointed rock samples subjected to cyclic triaxial loading. International Journal of Rock Mechanics and Mining Sciences, 98, 54-66. https://doi.org/10.1016/j.jijmms.2017.07.005

[4] Yi Liu, Feng Dai, Lu Dong, et al. (2018). Experimental Investigation on the Fatigue Mechanical Properties of Intermittently Jointed Rock Models under Cyclic Uniaxial Compression with Different Loading Parameters. Rock Mechanics and Rock Engineering, 51(1), 47-68. https://doi.org/10.1007/s00603-017-1327-7

[5] Debasis Deb \& Kamal Ch Das. (2009).Extended finite element method (XFEM) for analysis of cohesive rock joint. J. Sci. Ind. Res., 68, 575-583.

[6] Zhang, X. P. \& Wong, L. (2013). Crack initiation, propagation and coalescence in rock-like material containing two flaws: a numerical study based on bonded-particle model approach. Rock Mech. Rock. Eng., 46, 1001-1021. https://doi.org/10.1007/s00603-012-0323-1

[7] Wasantha P. L. P., Ranjith P. G., Viete, D. R. et al. (2012). Influence of the geometry of partially-spanning joints on the uniaxial compressive strength of rock. International Journal of Rock Mechanics and Mining Sciences, 50, 140-146. https://doi.org/10.1016/j.jirmms.2012.01.006

[8] Darot, M. \& Reuschlé, T. (2000). Acoustic wave velocity and permeability evolution during pressure cycles on a thermally cracked granite. International Journal of Rock Mechanics and Mining Sciences, 37(7), 1019-1026. https://doi.org/10.1016/S1365-1609(00)00034-4

[9] Song, I., Suh, M., Woo, Y. K., et al. (2004). Determination of the elastic modulus set of foliated rocks from ultrasonic velocity measurements. Engineering Geology, 72(3), 293308. https://doi.org/10.1016/j.enggeo.2003.10.003

[10] McCann, C. \& Southcott, J. (2009). Sonic to ultrasonic Q of sandstones and limestones: Laboratory measurements at in situ pressures. Geophysics, 74(2), 93-101. https://doi.org/10.1190/1.3052112

[11] Kurtulus, C., Üçkardes, M., Sari, U. et al. (2012). Experimental studies in wave propagation across a jointed rock mass. Bulletin of Engineering Geology and the Environment, 71(2), 231-234. https://doi.org/10.1007/s10064-011-0392-5

[12] Adam, L., Wijk, K. V., Otheim, T., et al. (2013). Changes in elastic wave velocity and rock microstructure due to basalt $\mathrm{CO}_{2}$ - water reaction. Journal of Geophysical Research-Solid Earth, 118(8), 4039-4047. https://doi.org/10.1002/jgrb.50302

[13] Yang Xiaohua, Yao Weixing, \& Duan Chengmei. (2003). The Review of Ascertainable Fatigue Cumulative Damage Rule. Engineering Science, 5(4), 81-87. (in Chinese)

[14] Zhu Jinsong \& Song Yupu. (2004). Research on Fatigue Damage of Concrete Under Biaxial Compressive Loading Using Ultrasonic Velocity Method. Chinese Journal of Rock Mechanics and Engineering, 23(13), 2230-2234. (in Chinese)

[15] Ge Xiurun. (1987). Study on Deformation and Strength Behaviour of the Large-sized Triaxial Rock Samples under Cyclic Loading. Rock and Soil Mechanics, 8(2), 11-19. (in Chinese)

\section{Contact information:}

Run LI, Corresponding author

Engineering College, Fujian Jiangxia University,

No. 2 Xiyuangong Road, University Town

Fuzhou, Fujian 350108, China

E-mail: Ir52tkd25@126.com

\section{Yasi WANG}

Engineering College, Fujian Jiangxia University, No. 2 Xiyuangong Road, University Town

Fuzhou, Fujian 350108, China

\section{Fengfei FU}

Engineering College, Fujian Jiangxia University, No. 2 Xiyuangong Road, University Town

Fuzhou, Fujian 350108, China 